\title{
Self-Monitoring in Social Networks
}

\author{
Amin Anjomshoaa ${ }^{1}$, Khue Vo Sao ${ }^{1}$, Amirreza Tahamtan ${ }^{1}$, \\ A Min Tjoa ${ }^{1}$, Edgar Weippl ${ }^{2}$ \\ ${ }^{1}$ Institute of Software Technology and Interactive Systems, Vienna University of Technology \\ 2 SBA-Research, Vienna, Austria \\ \{anjomshoaa, saokhue, tahamtan, amin\}@ifs.tuwien.ac.at; eweippl@sba-research.org
}

\begin{abstract}
Web 2.0 has changed the technological landscape of the Internet computing world today. The shift from traditional web which is also known as Web 1.0 is forced by the growing need for more efficient information sharing, collaboration, and business processes. The disclosure of personal/organizational information in Web 2.0 via social networks, digital contributions and data feeds has created new security and privacy challenges. Designing transparent, usable systems in support of personal privacy, security, and trust, requires advanced knowledge retrieval techniques that can support information sharing processes by applying appropriate policies. This paper proposes a Web 2.0 analysis methodology that provides reusable foundation components for information extraction, analysis and visualization. These components can be used by the same method can be also used by individuals to make a self-test of their Web 2.0 contributions and find out what inferences will be derived from their web presence.
\end{abstract}

Keywords: Web 2.0, Security and Privacy, Knowledge Retrieval

\section{Introduction}

Web 2.0 has made content creation much easier and as a result a huge amount of data is created by people every day. The volume of the data on the web is doubled since the emergence of Web 2.0 technologies. The data mining in the user generated entities and extracting the derived knowledge and information patterns is the new threat to privacy of individuals. We need more elaborate methods for analyzing the web contents in order to facilitate data sharing and data reuse in a trustworthy and efficient way. Moreover targeted data mining in the web data might be helpful in some use cases such as inter-organization trust.

Back in 1967, Doug Engelbart coined the collective IQ of organizations and of society as a grand challenge and the Web 2.0 is an attempt to come a step closer to this vision. It is believed that central principle behind the success of the giants born in the Web 1.0 era who have survived to lead the Web 2.0 era appears to be this, that they have embraced the power of the web to harness collective intelligence (O'Reilly, 2005).

One of the simplest and most common approaches for collective intelligence is the full-text search methods which allow people to query large data set using some key words. The query results are ranked according to some criteria such as frequency of key words in target resource and/or trustworthiness of the resource publishers. Algorithms for full-text searches are among the most important collective intelligence algorithms and a deciding factor for the success of search engines. For instance the rapid rise of Google from an academic project to the world's most popular search engine was based largely on their specific PageRank algorithm (Media, 2007). Despite all advantages of full-text search engines and their great contribution to information retrieval, in many cases the results are not relevant. For instance if you 
search any of the famous search engines for terms "Semantic Web" and "Architecture", the high rank results are mostly about "architecture of Semantic Web technology" which is misleading for a person who was looking for applications of

Semantic Web in Architecture, Engineering and Construction (AEC) field. This misunderstanding is because of the different applications of term architecture in different fields and the fact that computers deal with concepts as string of bytes.

One of the important outcomes of Web 2.0 is a large collection of semi-structured data that are used to empower the collective knowledge systems. The quality of such collective knowledge gets better as more people participate in data creation process and their contributions improve the quality of information. A good example for such collective knowledge system is the Wikipedia project. The database size of Wikipedia is increasing exponentially and many pages are being added each day.

On the other hand, despite critics on quality of Wikipedia articles, there are indicators that show the articles are getting more reliable. It is inherent in Wikipedia's editing model that poor information can be added, but over time quality is anticipated to improve in a form of group learning as editors reach consensus, so that substandard edits will very rapidly be removed. For example, the following table shows the number of Wikipedia citations in scientific journal articles indexed in ScienceDirect (Wikipedia, 2009):

Table 1. Number of research papers citing Wikipedia

\begin{tabular}{ll}
\hline Year article published & No. of articles citing Wikipedia \\
\hline Before 2003 & 0 \\
2003 & 1 \\
2004 & 9 \\
2005 & 28 \\
2006 & 129 \\
2007 & 358 \\
2008 (as of August 11 ) & 490 \\
\hline
\end{tabular}

The Web 2.0 approach for creating data has made the created data more structured in comparison to traditional web contents. For instance the taxonomy of weblog entries consists of title, description, creation date, author name, tags, and some comments; however this structure is not enough to make the content machineinterpretable. The current approaches to deal with knowledge extraction scenarios fall under two major research areas namely:

- deep syntactic and semantic analysis of human language

- automatic extraction of semantic relations from the text

The first method which has been a fundamental challenge for many years is still a complex task and many applied computational linguists turned to easier challenges such as text classifiers, text-to-speech converters, grammar checking and statistical machine translation. The second group of research activities is focusing on extraction of semantic relation of text and its context. The semantic relations cover a broad range of entities such as events, properties of objects, or geo temporal information that may be used to conclude a semantic understanding of the texts. 
The proposed methodology of this paper benefits from the advantages of the both text analysis methods and combines the results with the taxonomy of Web 2.0 applications. So on one hand there will be a rather comprehensive understanding of the text and on the other hand a taxonomy of different applications of Web 2.0 will help to join and unify this result for the sake of more complex processes such as security and privacy scenarios. Section 2 introduces the state of art in the context of classification and analysis of Web 2.0 contents. Section 3 describes our proposed methods to analyze user-created contents and visualize the information space of user on social networks.

\section{State of Art}

There are several methods for classification and analysis of Web 2.0 contents. One of the classification methods that has been widely used is tagging (Smith, 2007). The tag's quality improves as number of people that are tagging the same item increases.

Some Web 2.0 systems use the tag statistics and suggest the recommended tags to end user (Wetzker et al., 2008, Huang et al., 2010). It is important to note that such systems have no idea what the tags mean. The tag count and matching is done by system; however the tag semantics resides in the users' mind. In other words the linguistic meanings of the items are not injected into the machine via tagging. One possible approach for capturing the meaning of entries is mapping the tag tokens to an upper ontology that defines and connects the domain concepts. This can be done either by the text analysis or text matching techniques over the assigned tags.

A more elegant and more precise approach for enriching the Web 2.0 content is to embed the semantic information in the web content at creation time so that machines can read and interpret the content without the overhead of natural language processing methods. One of the solutions for making the web contents more intelligent is the W3C's initiative RDFa (W3C, 2008b) which provides a set of HTML attributes to augment visual data with machine-readable hints. It is highly beneficial to express the structure of web data in context; as users often want to transfer structured data from one application to another, sometimes to or from a non-web-based application, the user experience can be enhanced. For example, information about specific rendered data could be presented to the user via right-clicks on an item of interest (W3C, 2008a). There are many major use cases where embedding structured data in HTML using RDFa provides significant benefit (W3C, 2007). For example people's contact information, events and content's license such as creative commons can be included in web contents using RDFa syntax and relevant namespaces.

The RDFa is not the only solution for providing more intelligent data on the web. A similar approach for embedding machine-readable data in web content is microformats (Microformats, 2007) which is supposed to be coinciding with the design principles of "reduce, reuse, and recycle". The main difference between these approaches has historical background. The microformats have grown out of the work of blog developer community as an easy and ad-hoc response to common applications, but RDFa, on the other hand, is built with a more systematic vision of the W3 Semantic Web group and its associated thinkers. 
Unfortunately the content description methods are not being used by all content owners and the Web 2.0's Achilles' heel in our belief is the lack of semantic information that can be used to link this huge amount of information efficiently and this is the reason that some web specialists are expecting another web which is called Web 3.0 to complete the deficiencies of the current web. Without the explicit semantic context, the process of data analysis and putting the data to work in a business process safely is still unthinkable without significant human involvement and exactly here is the point that Semantic Web can fit, to make the data machineprocessable.

As a result the analysis of Web contents should be followed by other shallow knowledge extraction methods in order to add semantic annotations to the text. One of the well known challenges in this context is automatic Word Sense Disambiguation (WSD) for the given text.

WSD has been considered as a fundamental research problem in machine translation (Weaver, 1949) and artificial intelligence (Mallery, 1988) fields. WSD is the task of determining the sense of an ambiguous word in a given context of surrounding words, phrases, and sentences. For instance the word "library" can be understood as "library building" or "software library" according to its context and surrounding information. Fortunately the senses of English words can be easily extracted from free lexicon dictionaries such as WordNet (Princeton, 2010). Words in WordNet are organized in hierarchy and semantically instead of alphabetically. Each node consists of a synset of words, that express the same or a closely related word. These synsets are linked together by semantic relations such as hypernymy, hyponymy (nouns and verbs), meronymy (nouns), and antonymy (adjectives). Figure 1 , demonstrates some synsets (a group of related words that have the same gloss) and the semantic relations between them.

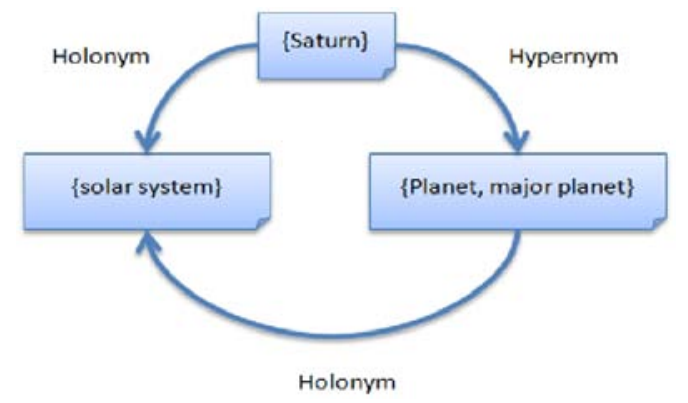

Fig. 1. WordNet semantic relations of some synsets

\section{Use Case Scenarios}

The Web 2.0 knowledge extraction and its applications demonstrate the power of collaborative work and how it can be used to assess collective data resources. The quality of such data resources is being improved via statistical and analytical methods of community behaviors (human oriented). An interesting point in such collective 
knowledge systems is vertical view that brings together the contributions of a specific user/organization and makes inferences about the behavior of user/organization. This vertical view which is also referenced as Gigantic Join, bring lots of benefits for Web

2.0 and Mashup architecture but on the contrary side threats the user's privacy by disclose the inferred facts about an individual or organization. For instance the vertical view of a person who publishes Youtube videos plus his/her social network might trigger a false positive alarm for relevant supervisors. There are two major concerns in such binary classifications:

- First of all it is important to note that the ratio of an out-of-favor group (such as terrorists, child abusers, etc) to the normal people is very small. So if the sensitivity (the proportion of people that tested positive of all the positive people tested) of classifier is selected to be very high there will be lots of false positives and consequently many normal people will be classified as out-of-favor and specificity (the proportion of people that tested negative of all the negative people tested) decreases.

- Another important issue is the fact that as soon as a person is incorrectly blacklisted (false positive) it would be very difficult (if not impossible) to removing him/her from the list. In other words there is a greater tendency to put the people in the black list rather than removing them from the list.

In order to hinder such scenarios, we would need an effective mechanism that can bridge the gap between information domains and aggregate value from a mix of structured and unstructured data. Due to the high degree of sensitivity that is used these days for classifiers, it looks essential to have a self-judgment tool that can be used by individual/organizations to correct their facade to the public and disable incorrect interpretations about themselves.

The knowledge extraction from Web 2.0 entries can be of great importance in many cases. In order to clarify the applications, we will discuss a number of such use cases in this section.

One of the interesting applications of content analysis is assistive services. The specific Web 2.0 contents should provide assistance for users who create similar contents. In other words, by analysis of existing contents, some targeted templates and information structure patterns will be established. These templates and patterns will be used to provide ad-hoc suggestions for common contents and structures for the given context. It is important to note that the assistive services are not allowed to share sensitive data with other users, instead they would share the templates and general structure of information. A good example for this group of services is the bookmark annotations that can be suggested to users according to the most favorite tags of a specific web page.

Another use case in knowledge extraction is the resource sharing. In Web 2.0 communities and especially in social networks, some information is being shared with other users. The "data sharing" in these environments is decided by content owner and there is no holistic solution to avoid the unwanted information disclosure. There is an ever growing need for intelligent sharing of the information based on the content of shared item, users' relationships, type of users, and personal-organizational sharing policies. 
The other group of use cases which are the center of attention in this paper is selfmonitoring of trust level. The data contributed by users on Web 2.0 is a good resource that can reflect the individual/organizational behaviors and attitude. So for example the membership in social networks, user groups, contributions on Wikipedia, FaceBook profile and friend network, blog entries, shared videos and pictures, and virtual games, are all together a rich set of information that can be used to judge about people or organizations. In some cases these inferences are not correct and the individuals and organizations have no mean to prevent false judgments. To address this issue there are some technical and social issues that will be discussed in the following sections.

All above mentioned scenarios have a common basic requirement, namely the automatic conceptualization of the targeted contents. Subsequently the conceptualized results can be used to provide assistive services, facilitate the resource sharing or in a higher scale, get combined with other data resources and used for self-monitoring purposes.

In our approach, we have tackled the automatic conceptualization challenges of Web 2.0 content by applying Semantic Web technologies. The outcomes of this step will provide a solid basis for addressing the assistive services and resource sharing scenarios. As a proof of concept the results are applied to the MindMeister framework (MindMeister, 2010) and show the feasibility of the first two use cases. Furthermore the self-monitoring scenario and the possible solution and drawbacks are explored and applied to user context in FaceBook social network (Facebook, 2010a).

\section{Our Solution}

Our approach can be summarized as follows:

1. The data will be extracted from social web platforms either by an API of target platform (such as FaceBook API (Facebook, 2010b), etc) or via a dedicated extractor component

2. Text analysis techniques such as WSD are applied to the text to disambiguate and annotate the text with useful semantic information

3. Self Organizing Maps (SOM) (Kohonen, 2001) are used to visualize the result and give the user an overview of his/her social network context.

4. After this step we apply quality measures to find out the high quality entries that have the potential for being used as template for assistive services. We have used this approach for MindMeister entries via the innovative quality measure that will be discussed in next section.

5. Another outcome of the SOM, are self-monitoring results by applying the user-ethical requirements and highlighting the points of interest on the resulting SOM in a given context.

The following figure demonstrated different steps of our solution to address the requirements of proposed use cases in previous section. 


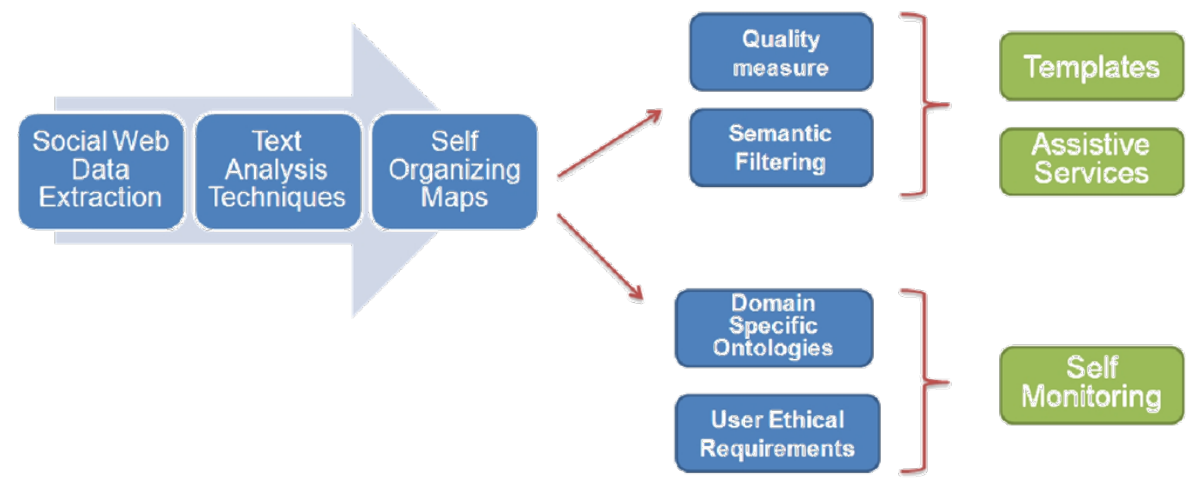

Fig 2. Overall solution for self-monitoring in Social Network

In the rest of this section each of the above mentioned steps will be discussed in more detail.

The first step toward using Web 2.0 contents in proposed use cases is to analyze the contents and extract the meaning out of them automatically. In this context the Web 2.0 API of the systems under study provide the required feeds for the text analysis component. There are a handful of techniques and algorithms for text analysis and information retrieval (IR) purposes (Doyle Lauren, 1975, Frakes, 1992). It goes without saying that the primitive methods such as stopping and stemming which are very standard in NLP and IR are not enough for the use cases of this proposal. Some other common techniques in this context are Chunk Parsing, Part Of Speech (POS) Tagging and Semantic Tagging.

In our approach we have used the SOM that is a powerful method for automatic clustering of high-dimensional statistical data in a way that similar inputs are mapped close to each other. Owing this property, the SOMs can be visualized easily and physical distance between concepts will depicts the similarity of concepts with regards to some predefined features of items in the domain under study.

A typical SOM algorithm for classification of text based items can be summarized as follows (Chen et al., 1996):

1. Initialize input nodes, output nodes, and connection weights: Use the top (most frequently occurring) $\mathrm{N}$ terms as the input vector and create a two-dimensional map (grid) of $\mathrm{M}$ output nodes. Initialize weights $\mathrm{w}_{\mathrm{ij}}$ from $\mathrm{N}$ input nodes to $\mathrm{M}$ output nodes to small random values.

2. Present each document in order: Describe each document as an input vector of $\mathrm{N}$ coordinates. Set a coordinate to 1 if the document has the corresponding term and to 0 if there is no such term. Each document is presented to the system several times.

3. Compute distance to all nodes: Compute Euclidean distance $\mathrm{d}_{\mathrm{j}}$ between the input vector and each output node $\mathrm{j}$ :

$$
d_{j}=\sum_{i=0}^{N-1}\left(x_{i}(t)-w_{i j}(t)\right)
$$


where $\mathrm{x}_{\mathrm{i}}(\mathrm{t})$ can be 1 or 0 depending on the presence of $\mathrm{i}$-th term in the document presented at time $\mathrm{t}$. Here, $\mathrm{w}_{\mathrm{ij}}$ is the vector representing position of the map node $\mathrm{j}$ in the document vector space. From a neural net perspective, it can also be interpreted as the weight from input node $i$ to the output node $j$.

4. Select winning node $j^{*}$ and update weights to node $j^{*}$ and its neighbors: Select winning node $j^{*}$, which produces minimum dj. Update weights to nodes $\mathrm{j}^{*}$ and its neighbors to reduce the distances between them and the input vector $\mathrm{xi}(\mathrm{t})$ :

$$
w_{i j}(t+1)=w_{i j}(t)+\eta(t)\left(x_{i}(t+1)-w_{i j}(t)\right)
$$

After such updates, nodes in the neighborhood of $\mathrm{j}^{*}$ become more similar to the input vector $x_{i}(t)$. Here, $\eta(t)$ is an error-adjusting coefficient $(0<\eta(t)<$ 1) that decreases over time.

5. After the network is trained through repeated presentations of all documents, assign a term to each output node by choosing the one corresponding to the largest weight (winning term). Neighboring nodes which contain the same winning terms are merged to form a concept/topic region (group). Similarly, submit each document as input to the trained network again and assign it to a particular concept in the map.

It is important to note that, similar items are clustered together if they share similar concepts and some concepts may carry more weight as compared to other concepts that appear in the same item, based on the concept frequency in that at item.

Since the SOM, cluster the words according to statistical methods, the semantic of words in their context is not considered. As a result, in order to improve quality of SOM, we need to disambiguate the concepts first. In other words, the WSD techniques are used to distinguish between different senses of the same word. For instance the term "library", can be either a building or a software library. By considering the context of this term and applying WSD techniques, this word can be clustered together with other relevant terms.

Nearly all WSD methods require some common preprocessing step that parses the input text and prepares it for further method-specific processes. These common preprocesses include actions such as tokenizing, stemming, and finding the meaning of the words in dictionaries or lexicons such as WordNet.

There are numbers of WSD methodologies for concluding the sense of words in a given context. One group of such methodologies relies mainly on gloss-based and path-based calculations. These methods which are also referred to as Lesk-based methods are listed below:

- $\quad$ Lesk algorithm (Lesk, 1986) disambiguates sense of the words by finding their gloss in traditional dictionaries and calculating its overlap with the glosses of its surrounding words.

- Kilgarriff \& Rosensweig (Kilgarriff and Rosenzweig, 2000) applied the original Lesk and considers also the the overlaps between a word's gloss with its context instead.

- Banerjee and Pederson (Banerjee and Pedersen, 2003) applied the original Lesk algorithm and used the WordNet instead of traditional dictionaries. 
Some other measures which are categorized as Semantic-based approaches, compute the path distance between two concepts based on the semantic organization of corresponding words in well-known taxonomies such as WordNet, in order to detect the sense of a given word. Some of the methods that take this approach are listed below:

- $\quad$ Leacock \& Chodorow (Leacock and Chodorow, 1998) measure the similarity of word senses based on their shortest path and their maximum depth of WordNet taxonomy in which similarity score is calculated as $-\log ($ length/ $2 * \mathrm{~d})$ where :

- $\quad$ length is the shortest path between two concept c1, c2 (relation "is a ") and

- $\quad d$ is the maximum depth of the taxonomy

- Wu \& Palmer (Wu and Palmer, 1994) method that measures the depth of the two concepts in WordNet taxonomy, and the depth of least common subsumer (LCS), and finally combines these figures into a similarity score as $2 * \mathrm{~d}(\mathrm{lcs}) /[\mathrm{d}(\mathrm{c} 1)+$ $\mathrm{d}(\mathrm{c} 2)$ ] where:

- $\quad d($ lcs) is the depth of the least common subsumer (LCS) and

- $\quad d(c 1), d(c 2)$ are the depth of concept c1, c2 respectively

Among WSD techniques, the Lesk family of algorithms is very suitable for the sense disambiguation of single words but they are not that effective when applied to a larger window of words. In contrast, semantic similarity measures compute the distance between the hierarchies of related synsets in WordNet and provide better results.

We have used the concepts hierarchy and their weights to align the ontology with domain ontologies, calculation of neighborhoods and semantic distance of different resources.

Since the proposed SOM method should be applied to a huge number of documents the traditional self organizing algorithm is not directly usable. Instead we will use a Scalable version of SOM (SSOM) (Roussinov and Chen, 1998), where data structure and algorithm took advantage of the sparsity of coordinates in the document's input vector and reduced the SOM computational complexity by several order of magnitude.

Another challenging issue is to take the extra information of Web 2.0 entries into consideration throughout the document processing phase. As mentioned before the Web 2.0 entries might have extra semi-structured information such as tags, attachments, relations, etc. This information can play an important role in the item analysis of Web 2.0 entries and should be extracted and included in the input vector of the SOM algorithm (step 1).

At the end of SOM process we will end up with a group of data points that are merged together and form clusters. The clusters are already labeled in step 5 and this labeling might be further improved in the next step for creating the ontologies.

An important note at this step is about security and privacy issues. After analysis of documents the sensitive content of the nodes should be removed according to domain ontology.

After creating SOM, we need to specify the points of interest on the map. In our approach, the points of interest are those areas that are violating the user ethics. User ethics can be classified as follows:

- Job ethics

o Professions have some ethical values that should be followed

o Organizations may also have some restrictions that should be followed by their employees

- $\quad$ Personal ethics

o People have their personal preferences and life styles

- Social ethics

o the community we are living in, may demand and require some social behavioral values 
These ethics are encoded in user profile, which can be combined with the semantic information of SOMs. For instance the job ethics might prohibit the user to share or contribute to discussions about a specific topic. In such cases the violating areas will be highlighted on SOM and user may react and take the necessary actions to correct the situation. The following figure, demonstrates this use case where high risk group of friends are highlighted on the interest map of friends.

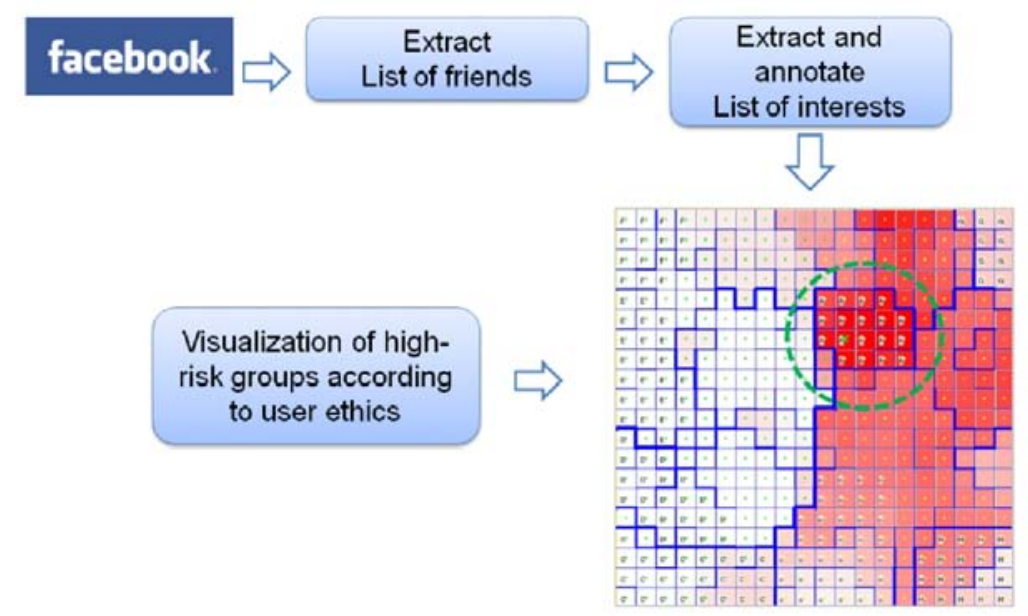

Fig 3. Visualization of high risk group on the friends' interest map

In order to facilitate user interactions with the developed components we are now planning to employ Mashup architecture for presenting the services which provide a user-driven micro-integration of web-accessible data. At the moment Mashups are mainly used for less important tasks such as customized queries and map-based visualizations; however they have the potential to be used for more fundamental, complex and sophisticated tasks in combination with business processes. One important benefit of the Mashup architecture is the possibility to reuse existing components and also share the community-generated components with other users, which might need it. This will radically decrease the required time of development and implementation phases.

Mashup solutions facilitate the collection, integration, and publishing of data for nonexpert users. As a result, the Mashup solutions can be seen as new data sources that may feed other Mashups, applications, services, or websites for business / personal use cases in an easy way. 
The following figure demonstrates a simple Mashup that use the available services of proposed solution, to create a SOM of all books that friends of a specific user are interested in. The map is then highlighted according to job ethics of interest book of this user, to find out the inappropriate connections in his/her FaceBook profile.

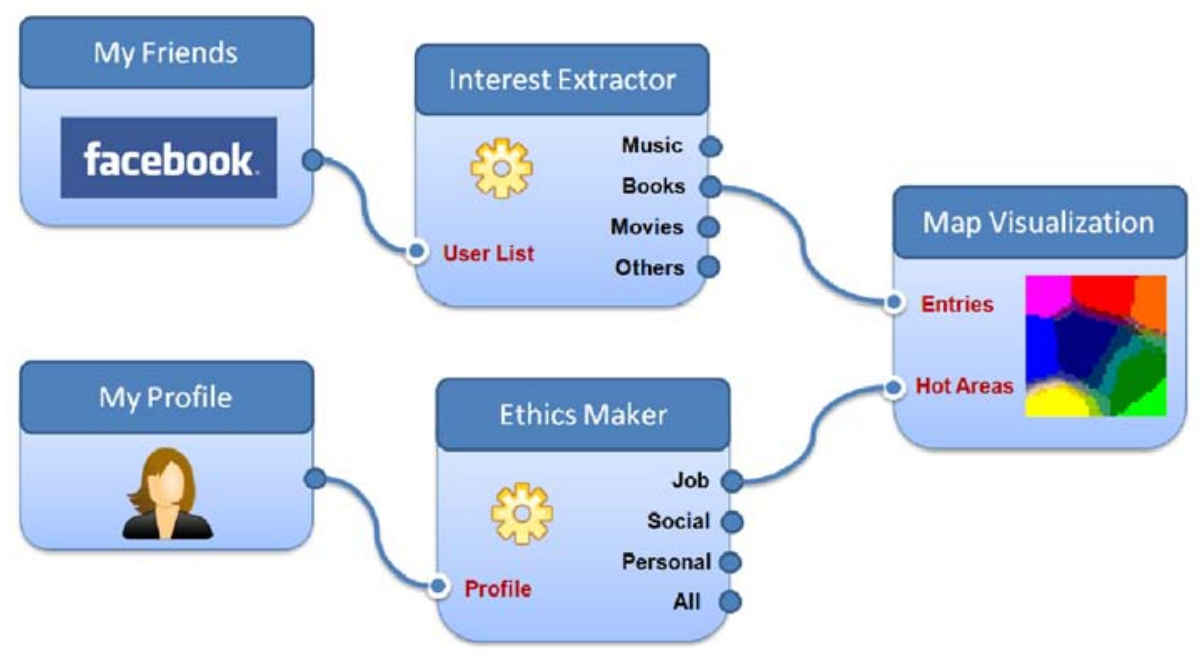

Fig 4. A Mashup outlook to create a SOM of friends' interest in Facebook

\section{MindMeister Case Study}

In this section a quality measure for specific case of mind maps (MindMeister entries) will be presented. MindMeisters entries are planned to be equipped with the following two groups of services:

- The first group of services should provide a higher level of trust in data sharing. Such services will take care of filtering of private and sensitive data and hinder the unwanted disclosure of such data based on some predefined data sharing policies.

- The second group of services is aiming to provide some assistive services that make the mind map creation easier and more efficient for end users. In order to create such assistive services, the repository of shared mind maps which includes thousands of user-contributed mind maps, should be analyzed and ranked based on quality of organized information. The high quality mind maps can then be transformed to mind map templates that facilitate creation of mind maps in diverse knowledge domains.

To address the requirement of these services, the mind map content and its data organization should be analyzed for interpreting its knowledge domain, the concepts it covers, and its information quality. The quality indicator of a mind map which is one of the main concerns of this paper, should determined quality of both its content and its information organization. The quality indicator of each mind map will be then 
used to select high quality mind maps that have the potential to form a goo template for diverse usages such as project management, human resource management, party organization, etc.

To clarify the issue consider the mind map that given in figure 5 , and demonstrates some concepts of our solar system. A rather better organization of these concepts is given in figure 6, where the planets are listed together under a specific node for planets. The ultimate goal of this paper is to provide a methodology to make this conclusion process machine-interpretable via appropriate quality indicators.

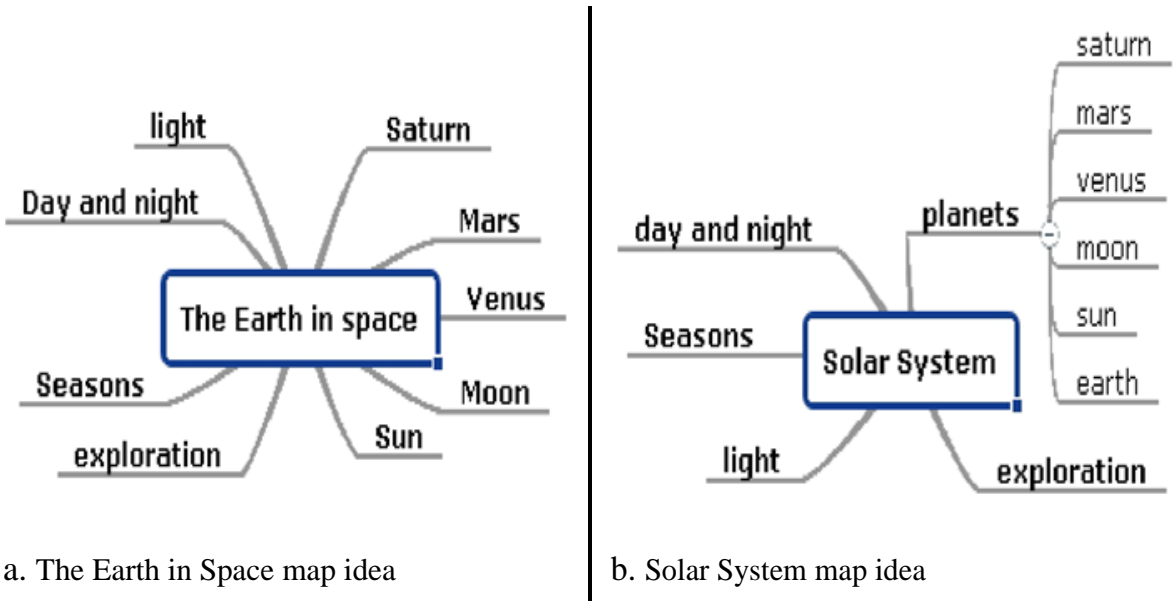

Fig. 5. The Earth in Space map idea in MindMeister

In this case study, we first use a hybrid approach to accomplish the WSD of a mind map. In the first step Lesk algorithm is used to find the correct sense of a word according to its context. A word's context in mind maps is assumed to be the text of parent node, the text of the node itself, and finally the accumulative text of all subnodes.

At the end of the WSD process the words will be annotated using the most appropriate sense of the word in the given context, based on WordNet senses.

Suppose $\left\{I_{a} \ldots I_{c}\right\}$ is the set of nodes (or Ideas in MindMeister terminology) in a given mind map that contains a set of words (concepts) $\left\{\mathrm{w}_{\mathrm{i}} \ldots \mathrm{w}_{\mathrm{j}}\right\}$, and each word has some senses with a specific SynsetID $\left\{\mathrm{s}_{\mathrm{i}} \ldots \mathrm{s}_{\mathrm{k}}\right\}$. The annotation tags of words will be then created by combining the words (stem of words) and the correct SynsetID. As a result all words will be uniformly annotated by a well-defined taxonomy.

Another challenging issue in our approach was that some words do not exist in lexicons. As an example the commercial or technical words such as Mashup, Flickr, or Servlet cannot be found in WordNet. To address this issue, such terms are queried via Google Search API (Google, 2010) and the results taking from Wikipedia will be considered as its gloss. In most cases, this approach will provide suitable definition for given terms. For instance, WordNet returns no results for the term "Mashup"; however the result from Wikipedia in Google searching says: "In web development, $a$ 
mashup is a web page or application that combines data or functionality from two or more external sources to create a new service. ...", which is very helpful for our purposes. The final output of this approach is an annotated mind map (annotated with correct sense of the words) that can be used for further knowledge extraction and analysis purposes and also an overall quality indicator that expresses the rationale and semantic quality of the given mind map. The rest of this section is dedicated to the required steps for creating a quality measure for mind maps. First of all we need to define a similarity score between two given words $\mathrm{W}_{\mathrm{a}}, \mathrm{W}_{\mathrm{b}}$. As explained before there might be some words that are not included in WordNet. In such cases the similarity measure will be defined as follows:

$$
\operatorname{sim}\left(\mathrm{w}_{\mathrm{a}}, \mathrm{w}_{\mathrm{b}}\right)=\left[\text { overlaps } /\left(\text { length }\left(\mathrm{G}_{\mathrm{a}}\right)+\text { length }\left(\mathrm{G}_{\mathrm{b}}\right)\right)\right]
$$

Where overlap is the length of overlapping sections in glosses of two words (taken from WordNet or Google Search API) and $G_{a}$ and $G_{b}$ are glosses of $W_{a}$ and $W_{b}$ respectively. For those cases that both words can be found in WordNet, the similarity measure will be defined as follows:

$\operatorname{sim}\left(\mathrm{w}_{\mathrm{a}}, \mathrm{w}_{\mathrm{b}}\right)=[$ common_nodes $] /\left[\right.$ shortest_path $\left.\left(\mathrm{w}_{\mathrm{a}}, \mathrm{w}_{\mathrm{b}}\right)\right]+$ synset_relation

where:

- common_nodes: is the depth of common nodes in WordNet hierarchy of the given word's senses.

- shortest_path: is the shortest connecting path between two synsets of wa, wb in WordNet.

- Synset_relation: is the measure that depicts if two synset have any semantic relationships such as hyponym, holonym, etc. In case such relationship exists the value of Synset_relation is "one" otherwise it is assumed to be "zero". The synset_relation value clearly specifies if the given nodes of mind map have any logical relationship and the mind map delivers a comprehensive idea. For instance the synset relation in figure 1 shows that the mind map of figure 5(b) is logically better than the mind map of figure 5(a).

The meaning of common_nodes and shortest_path are depicted in figure 6 . 


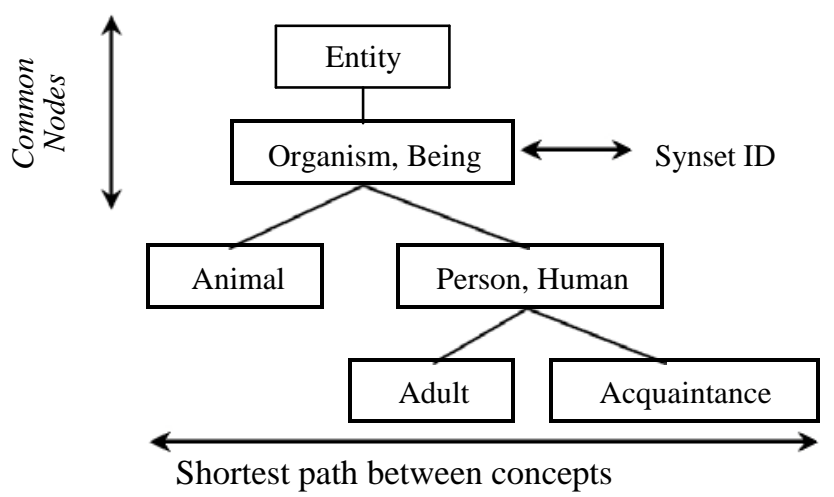

Fig. 6. Common nodes and shortest path between two sample concepts (Animal, Acquaintance)

In order to extend the introduced measure to mind map nodes (ideas), the following formula has been used:

$$
\operatorname{sim}\left(\mathrm{I}_{\mathrm{a}}, \mathrm{I}_{\mathrm{b}}\right)=\sum_{i=1}^{m} \sum_{j=1}^{n} \operatorname{sim}(W i, W j) /(\mathrm{m}+\mathrm{n}) .
$$

Where " $m$ " and " $n$ " are length of $I_{a}$ and $I_{b}$ respectively. This formula simple suggest that the similarity of two ideas can be calculated based on the similarity score of their cross products. This process will be done after initial cleansing in order to reduce the processing time. As the final step, the given mind map will be traversed and number of the ideas that are within a predefined threshold will be counted. This value which demonstrates the semantic degree of mind maps will be used as quality measure to rank the mind map clusters that are focused on specific topics such as project management, party organization, etc.

The result of this approach for case of the given mind maps in figure 5, specifies 0.20558557 as quality measure for figure 5(a) and 0.4570171 for figure 5 (b) which supports our hypothesis about semantic quality of these two maps.

\section{Results}

As proof of concept we have applied the proposed approach to Twitter and FaceBook use cases. In Twitter use case, the twits of a user are extracted from Twitter using Twitter API (Twitter, 2010). In the next step the words are disambiguated and visualizes in a SOM. In order to apply the SOM algorithm we would need an input vector of features. Since in Web 2.0 use cases such vector does not exist, we have used the top 1000 frequent words that have been occurring in the input twits. The result of this process is depicted in the following figure. 


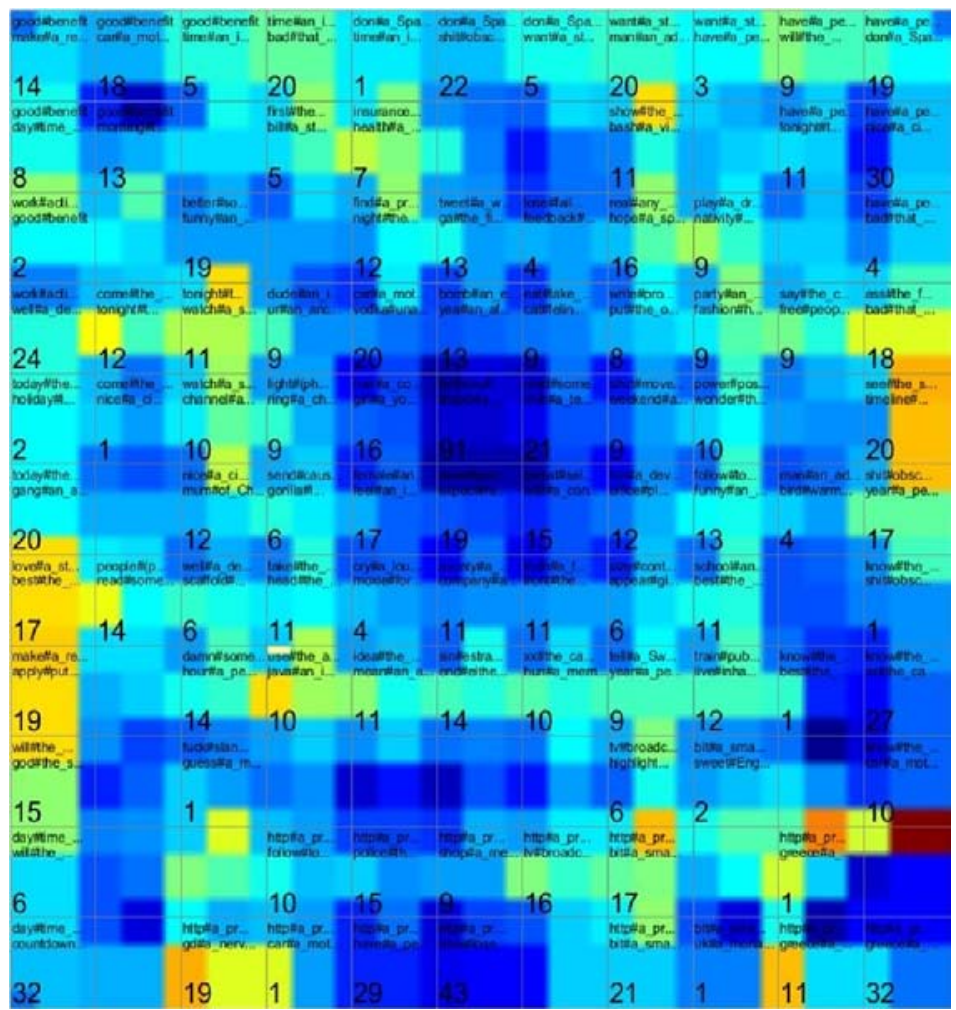

Fig. 7. SOM Visualization of Twitter twits

In the second use case, we have extracted the data from FaceBook API for a specific user. In FaceBook environment, each user may have a number of friends in his/her network, where each friend has a collection of interests including books, movies, music, etc. Many users may lose the overview of what their friend are doing and this may lead to incorrect judgments about them when the interest of a friend is out-of-favor. For this purpose, the user's network of friends is scanned and all interests will be extracted. In the next step, the interest items are automatically annotated with relevant information such as book category, film genre, etc. As the final step the items will be displayed on a SOM. The following figure shows the interest map of all friends for a specific user. In similar way, user will be able to go into more details and visualize the interest map of a single friend. 


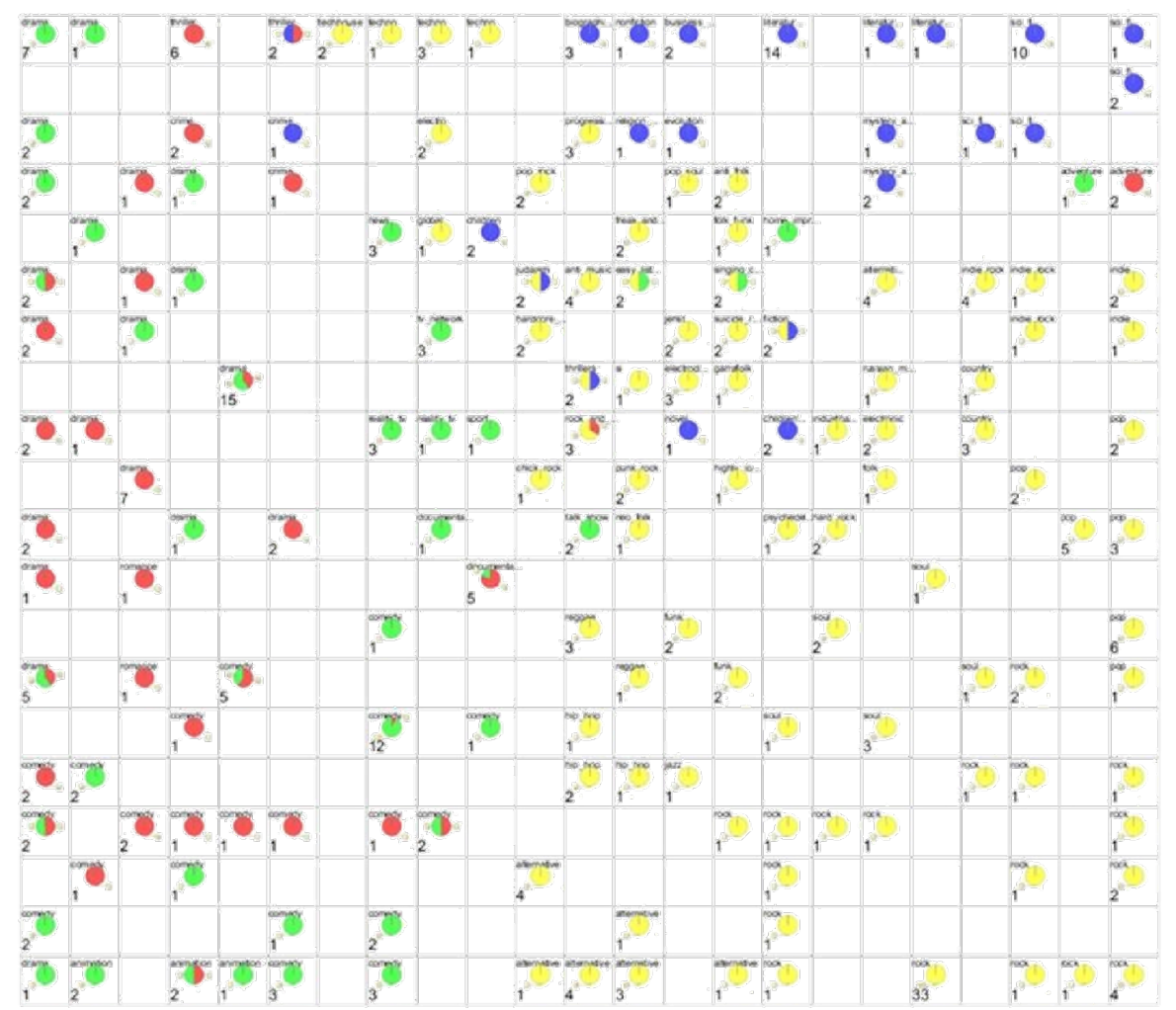

Fig. 8. SOM Visualization for friend's interest map of Facebook

\section{Conclusion}

The proposed methodology of this paper benefits from the advantages of text analysis methods and combines the results with the taxonomy of Web 2.0 applications. This methodology has been successfully applied to some real world use cases has delivered promising results. The proposed approach has also introduced reusable foundation components for knowledge extraction and data analysis that can be applied to different Web 2.0 entries.

We are now in the process of unifying the developed services as Mashup widgets that can be easily used by end users to monitor their presence on the web and improving their public image.

Acknowledgments. This research is supported by FIT-IT project, Secure 2.0 (project number: 820852). 


\section{References}

Banerjee, S. \& Pedersen, T. (2003). Extended gloss overlaps as a measure of semantic relatedness. Proceedings of the 18th international joint conference on Artificial intelligence. Acapulco, Mexico: Morgan Kaufmann Publishers Inc.

Chen, H., Schuffels, C. \& Orwig, R. (1996). Internet Categorization and Search: A Self-Organizing Approach. Journal of Visual Communication and Image Representation, 7, 88-102.

Doyle Lauren, B. J. (1975). Information Retrieval and Processing. Melville.

Facebook. (2010a). Facebook. Available: http://www.facebook.com.

Facebook. (2010b). Facebook API. Available: http://developers.facebook.com/.

Frakes, W. B. (1992). Information Retrieval Data Structures \& Algorithms. Prentice-Hall, Inc.

Google. (2010). Google Ajax Search API. Available: http://code.google.com/apis/ajaxsearch/.

Huang, J., Thornton, K. M. \& Efthimiadis, E. N. (2010). Conversational tagging in twitter. Proceedings of the 21st ACM conference on Hypertext and hypermedia. Toronto, Ontario, Canada: ACM.

Kilgarriff, A. \& Rosenzweig, J. (2000). Framework and Results for English SENSEVAL. Computers and the Humanities, 34, 15-48.

Kohonen, T. (2001). Self-Organizing Maps. Springer.

Leacock, C. \& Chodorow, M. (1998). Combining Local Context and WordNet Similarity for Word Sense Identification. An Electronic Lexical Database, 265-283.

Lesk, M. (1986). Automatic sense disambiguation using machine readable dictionaries: how to tell a pine cone from an ice cream cone. Proceedings of the 5th annual international conference on Systems documentation. Toronto, Ontario, Canada: ACM.

Mallery, J. C. (1988). Thinking about foreign policy: Finding an appropriate role for artificial intelligence computers. Ph.D Dissertation. MIT Political Science Department, Cambridge.

Media, O. R. (2007). Programming Collective Intelligence: Searching and Ranking, white paper. Available: http://whitepapers.techrepublic.com.com/abstract.aspx?docid=323363.

Microformats. (2007). Microformats. Available: http://microformats.org/.

MindMeister. (2010). MindMeister. Available: http://www.mindmeister.com/.

O'Reilly, T. (2005). What Is Web 2.0: Design Patterns and Business Models

for the Next Generation of Software. Available: http://www.oreillynet.com/pub/a/oreilly/tim/news/2005/09/30/what-is-web20.html.

Princeton, U. (2010). WordNet - a large lexical database of English. Available: http://wordnet.princeton.edu/.

Roussinov, D. \& Chen, H. (1998). A Scalable Self-organizing Map Algorithm for Textual Classification: A Neural Network Approach to Thesaurus Generation. Communication and Cognition, 15, 81-112.

Smith, G. (2007). Tagging: People-powered Metadata for the Social Web (Voices That Matter). New Riders Press.

Twitter. (2010). Twitter Developers. Available: http://dev.twitter.com/.

W3C. (2007). RDFa Use Cases: Scenarios for Embedding RDF in HTML. Available: http://www.w3.org/TR/xhtml-rdfa-scenarios/.

W3C. (2008a). RDFa in XHTML: Syntax and Processing. Available: http://www.w3.org/TR/2008/CR-rdfa-syntax-20080620.

W3C. (2008b). RDFa syntax. Available: http://www.w3.org/TR/2008/CR-rdfasyntax-20080620l.

Weaver, W. (1949). Translation. In: D., A. (ed.) Machine Translation of Languages ed. New York: John Wiley \& Sons.

Wetzker, R., Zimmermann, C. \& Bauckhage, C. Year. Analyzing Social Bookmarking Systems: A del.icio.us Cookbook. In: Proceedings of the 
ECAI 2008 Mining Social Data Workshop, 2008. IOS Press, 26-30.

Wikipedia. (2009). Reliability of Wikipedia. Available:

http://en.wikipedia.org/wiki/Reliability of Wikipedia.

Wu, Z. \& Palmer, M. (1994). Verbs semantics and lexical selection. Proceedings of the 32nd annual meeting on Association for Computational Linguistics. Las Cruces, New Mexico: Association for Computational Linguistics. 